\title{
Comment on "Puerarin Improves Diabetic Aorta Injury by Inhibiting NADPH Oxidase-Derived Oxidative Stress in STZ-Induced Diabetic Rats"
}

\author{
Qiang Xie, ${ }^{1}$ Jian Zhong, ${ }^{2}$ and Jun $\mathrm{Li}^{\mathbf{3 , 4 , 5}}$ \\ ${ }^{1}$ PET/CT Center, Anhui Provincial Hospital, Hefei 230001, China \\ ${ }^{2}$ Anhui Joyfar Pharmaceutical Co., Ltd., Kexuedadao Road, Hefei 230000, China \\ ${ }^{3}$ School of Pharmacy, Anhui Medical University, Meishan Road, Hefei 230032, China \\ ${ }^{4}$ Institute for Liver Diseases, Anhui Medical University, Hefei 230032, China \\ ${ }^{5}$ Anhui Key Laboratory of Bioactivity of Natural Products, Anhui Medical University, Hefei 230032, China \\ Correspondence should be addressed to Jun Li; lj@ahmu.edu.cn
}

Received 18 May 2016; Accepted 23 June 2016

Academic Editor: Hiroshi Okamoto

Copyright (C) 2016 Qiang Xie et al. This is an open access article distributed under the Creative Commons Attribution License, which permits unrestricted use, distribution, and reproduction in any medium, provided the original work is properly cited.

We read with great interest the article showing that streptozotocin- (STZ-) induced diabetic rats given puerarin can significantly reduce the serum levels of insulin, glycated hemoglobin, PGE2, endothelin, $\mathrm{H}_{2} \mathrm{O}_{2}$, and $\mathrm{NO}$, improve the pathological alterations, and inhibit the expression of ICAM-1, LOX-1, and NOX2 [1]. In addition, puerarin strongly reduced the number of cells showing positive staining for ICAM-1, NOX2, NOX4, and NF- $\kappa$ B p65. Interestingly, Li et al. also showed that STZ-induced diabetes mellitus and aorta lesion can be improved by treatment with puerarin, where puerarin is able to downregulate the levels of cholesterol, low density lipoprotein, P-selectin, oxidative low density lipoprotein, and the expression of VCAM in the aorta which were significantly inhibited [2]. These data indicated that puerarin has played a certain role in preventing aorta or vessels by inhibiting expression of adhesion molecules.

Diabetes is a chronic disease characterized by hyperglycemia. It results in numerous chronic microvascular and macrovascular complications, such as nephropathy and atherosclerosis. These chronic complications are the major causes of the downregulated quality of life among diabetics, elevated burden to the health care system. Thus, inhibiting and alleviating these complications is helpful in diabetes treatment. Puerarin is the main bioactive component of getongtongluo capsule, extracted from the Chinese herb lobed kudzuvine root [3]. Puerarin has been recognized to inhibit high glucose-induced upregulation of $\mathrm{H} 3 \mathrm{~K} 4 \mathrm{di}-$ and trimethylation ( $\mathrm{H} 3 \mathrm{~K} 4 \mathrm{me} 2 / 3)$ on the MCP-1 gene promoter, possessing a therapeutic potential in diabetes-induced vascular injuries [4]. It also significantly inhibited rat vascular smooth muscle cells proliferation, ROS generation, and NADPH oxidase activity induced by high glucose treatment [5]. Interestingly, neointimal formation of obese rats evoked by balloon injury was attenuated by the administration of puerarin. Moreover, insulin resistance was established by palmitate stimulation in the endothelium, and Huang et al. [6] showed that palmitate stimulation evoked inflammatory response in endothelial cells but puerarin inhibited IKK $\beta / \mathrm{NF}-\kappa \mathrm{B}$ activation and decreased TNF- $\alpha$, IL- 6 production, and insulin-mediated $\mathrm{NO}$ generation. In addition, rats incubated with high glucose decreased the vascular contraction responses to phenylephrine and relaxation response to acetylcholine, but treatment with puerarin inhibited the high glucose-induced vasoconstriction and vasodilation dysfunction and increased the $\mathrm{HO}-1$ protein expression and $\mathrm{HO}$ activity of thoracic aorta [7].

Collectively, the available evidence suggests a potential role that puerarin plays in aorta or vessels improvement, 
especially in diabetes. However, further studies are needed to comprehensively explore the role of puerarin in diabetes and its complications, and the application of these therapeutic agents should be discussed in humans though available evidence is mainly determined in animals.

\section{Competing Interests}

Jian Zhong is affiliated to Anhui Joyfar Pharmaceutical Co. Ltd., manufacturer of getongtongluo capsules that contain puerarin.

\section{References}

[1] W. Li, W. Zhao, Q. Wu, Y. Lu, J. Shi, and X. Chen, "Puerarin improves diabetic aorta injury by inhibiting NADPH oxidasederived oxidative stress in STZ-induced diabetic rats," Journal of Diabetes Research, vol. 2016, Article ID 8541520, 9 pages, 2016.

[2] Q. Li, X. Zhang, F. Zhu, and Z. Zhang, "Study of regulation of Puerarin on blood P-selectin and the expression of aorta VCAM mRNA in diabetes," Wei Sheng Yan Jiu, vol. 36, no. 5, pp. 581583, 2007.

[3] H. Li, K. Luo, and J. Hou, "Inhibitory effect of puerariae radix flavones on platelet-derived growth factor-bb-induced proliferation of vascular smooth muscle cells via pi3K and ERK pathways," Experimental and Therapeutic Medicine, vol. 9, no. 1, pp. 257-261, 2015.

[4] P. Han, D. Gao, W. Zhang, S. Liu, S. Yang, and X. Li, "Puerarin suppresses high glucose-induced MCP-1 expression via modulating histone methylation in cultured endothelial cells," Life Sciences, vol. 130, pp. 103-107, 2015.

[5] L.-H. Zhu, L. Wang, D. Wang et al., "Puerarin attenuates highglucose-and diabetes-induced vascular smooth muscle cell proliferation by blocking PKC $\beta 2 /$ Racl-dependent signaling," Free Radical Biology and Medicine, vol. 48, no. 4, pp. 471-482, 2010.

[6] F. Huang, K. Liu, H. Du, J. Kou, and B. Liu, "Puerarin attenuates endothelial insulin resistance through inhibition of inflammatory response in an IKK $\beta /$ IRS-1-dependent manner," Biochimie, vol. 94, no. 5, pp. 1143-1150, 2012.

[7] X.-H. Meng, C. Ni, L. Zhu, Y.-L. Shen, L.-L. Wang, and Y.-Y. Chen, "Puerarin protects against high glucose-induced acute vascular dysfunction: role of heme oxygenase-1 in rat thoracic aorta," Vascular Pharmacology, vol. 50, no. 3-4, pp. 110-115, 2009. 


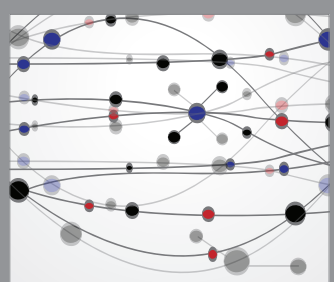

The Scientific World Journal
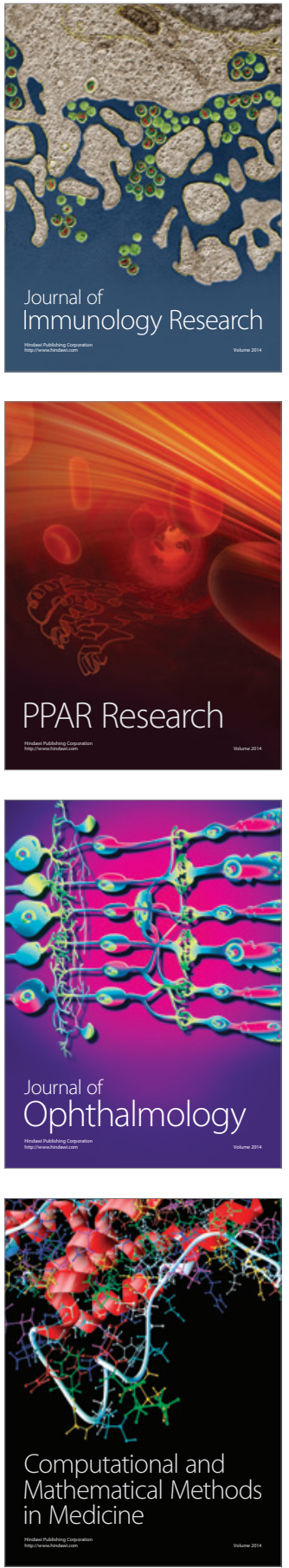

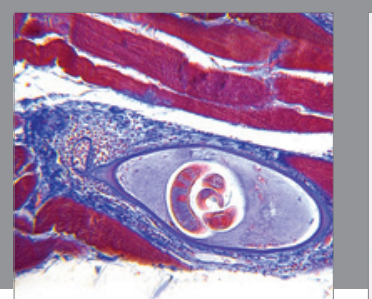

Gastroenterology Research and Practice

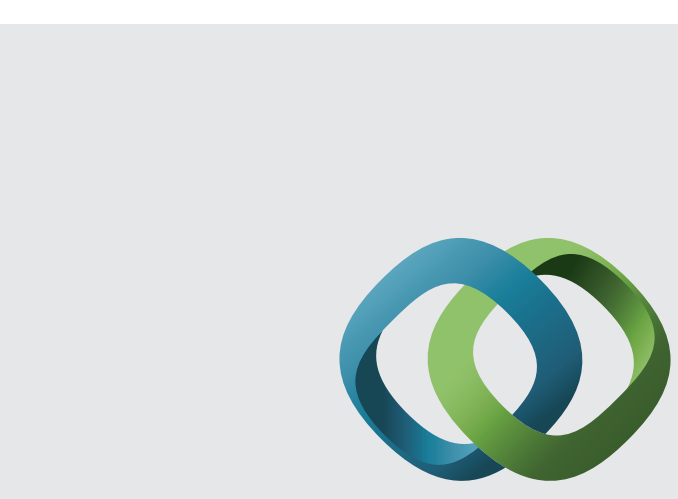

\section{Hindawi}

Submit your manuscripts at

http://www.hindawi.com
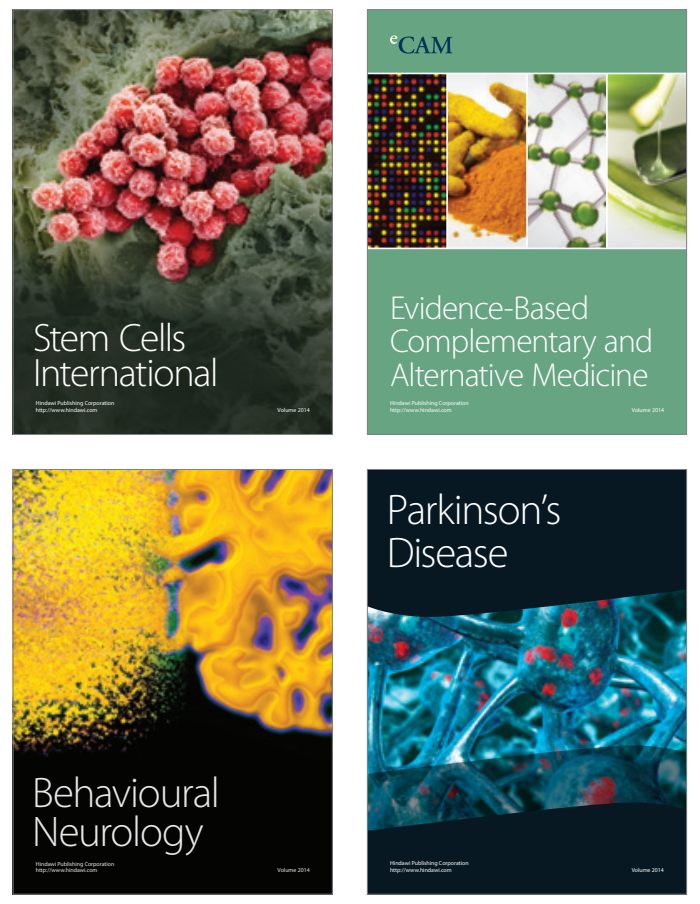
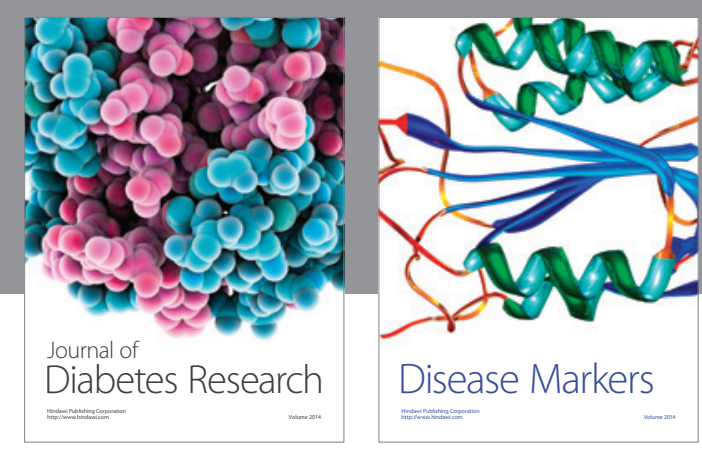

Disease Markers
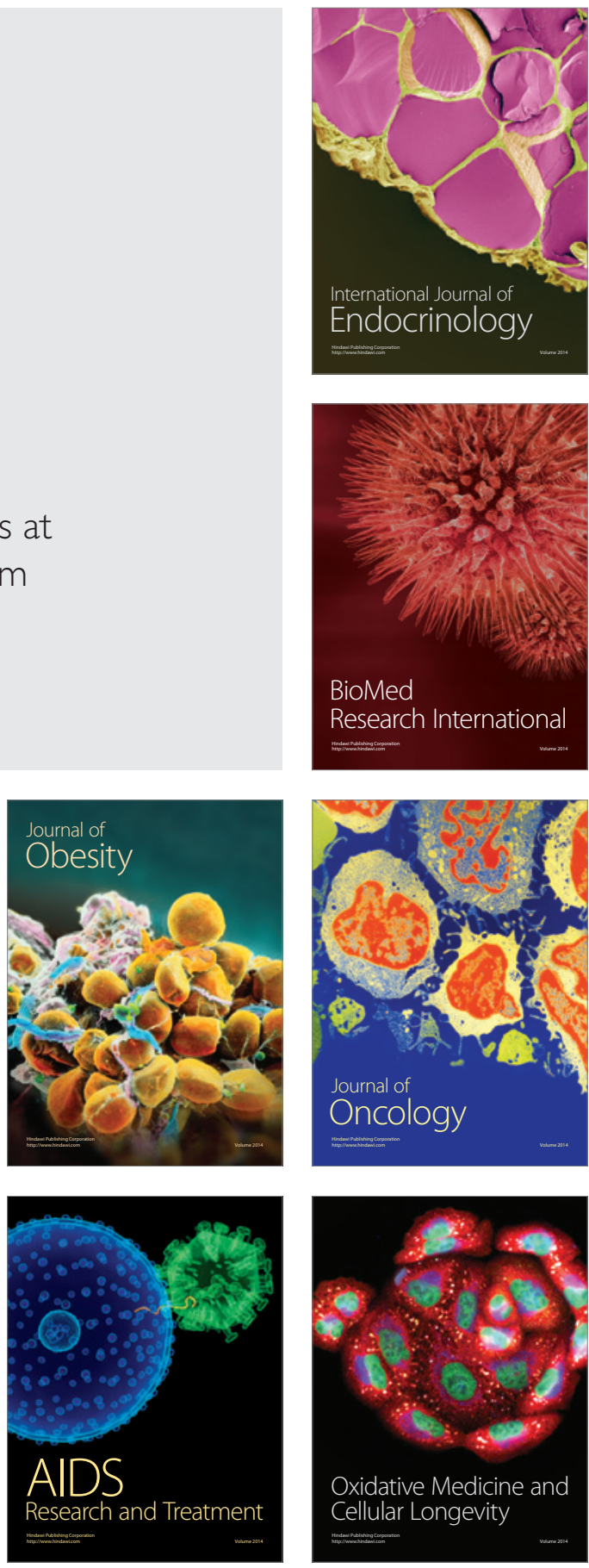